\title{
Association between sedentary behaviour and risk of dementia: an evidence gap
}

\author{
Kirsten Dillon $\mathbb{1}^{1}$ and Paul A. Gardiner (1) ${ }^{1,2}$
}

Dear Editor,

Given the lack of a cure, it is important to investigate risk factors for dementia to target prevention initiatives. A study recently published in Translational Psychiatry by Yan et al. ${ }^{1}$ examines the association between sedentary behaviour [sic] and the risk of dementia. However, the authors have misused the term sedentary behaviour, which misrepresents their findings and leads to a false conclusion. Historically, sedentary behaviour was used to denote lack of physical activity. Now 'sedentary behaviour' is defined as "any waking behaviour characterized by energy expenditure $\leq 1.5$ metabolic equivalents while in a sitting, reclining or lying posture" ${ }^{2}$. In contrast, the term 'physical inactivity' is defined as "an insufficient physical activity level to meet present physical activity recommendations" ${ }^{2}$. For example, the World Health Organization (WHO) states the global physical activity guidelines as $150 \mathrm{~min}$ of moderate-intensity, or $75 \mathrm{~min}$ of vigorousintensity aerobic physical activity a week (WHO, 2020 ${ }^{3}$. As many people in today's society are both physically inactive and sedentary ${ }^{4}$, it is imperative that we differentiate between these two terms.

Ten years ago, Owen and colleagues clearly defined and differentiated the various health consequences of too much sitting (sedentary behaviour) versus physical inactivity $^{5}$. In 2012, the Sedentary Behaviour Research Network (SBRN) was aware of the inconsistencies in the terminology around sedentary behaviour and proposed a formal definition of sedentary behaviour, which was updated in 2017 (see above). Given the increasing evidence on the deleterious associations of sedentary behaviour with health outcomes ${ }^{6}$, the Sedentary Behaviour Council of the International Society of Physical Activity and Health successfully advocated for the (United States)

\footnotetext{
Correspondence: Kirsten Dillon (kdillon9@uwo.ca)

${ }^{1}$ The University of Western Ontario, London, Canada

${ }^{2}$ The University of Queensland, Brisbane, Australia
}

National Library of Medicine to create a sedentary behaviour Medical Subject Heading (MESH) term ${ }^{7}$. Without the proper definition and use of these terms going forward, future research will be confused by their inappropriate use.

Sedentary behaviour research is well established with evidence accumulating for over two decades on deleterious associations with health outcomes such as a higher risk of type 2 diabetes, cardiovascular disease (fatal and non-fatal), and all-cause mortality ${ }^{6}$. More nascent evidence is emerging on the relationship of sedentary behaviour with cognitive function. Since 2016, there have been four systematic reviews published indicating mixed associations between various sedentary behaviours and cognitive function ${ }^{8-11}$. The first review by Falck et al. ${ }^{9}$ indicated that higher levels of sedentary behaviour are associated with lower cognitive function. Copeland et al. ${ }^{8}$ then indicated that the association may vary depending on the domain of sedentary behaviour being assessed. Finally, Loprinzi ${ }^{10}$ and Olanrewaju et al. ${ }^{11}$ stated an overall lack of clarity in the association of sedentary behaviour with cognitive function. Overall, although there was a slight trend towards more sedentary behaviour being associated with worse cognitive function, none of these reviews were able to distinguish a clear association of sedentary behaviour with the risk of dementia. Therefore, a gap remains, which was why we were encouraged to read this article by Yan et al. ${ }^{1}$. However, upon further investigation of the article, it became clear that it does not address this association, and thus, the gap still remains. More specifically, the article fails to properly define 'sedentary behaviour' as the exposure variable. As a result of this, none of the studies included within the systematic review and meta-analysis by Yan et al. ${ }^{1}$ were in fact reporting 'sedentary behaviour' as the exposure variable but rather physical inactivity (see Table 1). Hence, it is recommended that the authors revise their manuscript to 
Table 1 Description of exposure variables used within each study of Yan et al.'s ${ }^{1}$ meta-analysis.

\begin{tabular}{|c|c|}
\hline Study & Exposure variable \\
\hline Deckers et al. (2018) & Physical inactivity: No exercise or physical activity in the last week. \\
\hline Kishimoto et al. (2016) & Physical inactivity: No reported leisure time exercise in the last week. \\
\hline Luck \& Riedel-Heller (2016) & Physical inactivity: Proportion of the adult population that self-report not participating in sports. \\
\hline MacDonald et al. (2015) ${ }^{a}$ & Physical inactivity: No definition. \\
\hline Luck et al. (2014) & Physical inactivity: Participating in an activity less than or equal to once a week, or never. \\
\hline Mehlig et al. (2014) & Physical inactivity: Less than $4 \mathrm{~h}$ of leisure time physical activity. \\
\hline Norton et al. (2014) & $\begin{array}{l}\text { Physical inactivity: Do not do either } 20 \text { min of vigorous activity on } 3 \text { or more days or } 30 \text { min of moderate activity on } 5 \text { or } \\
\text { more days per week. }\end{array}$ \\
\hline de Bruijn et al. (2013) & $\begin{array}{l}\text { Physical activity: Hours spent on activities such as walking, cycling, gardening, diverse sports, hobbies and housekeeping } \\
\text { activities. }\end{array}$ \\
\hline Verdelho et al. (2012) & Physical inactivity: Not participating in at least 30 min of activity on at least 3 days per week. \\
\hline Gelber et al. (2012) & $\begin{array}{l}\text { High physical activity: Defined as the highest quartile of daily hours spent in slight or moderate activity (e.g., walking, } \\
\text { gardening, carpentry). } \\
\text { Low physical activity: Sleeping, lying, sitting or standing. }\end{array}$ \\
\hline Keller et al. (2010) ${ }^{a}$ & Physical inactivity: No activity with a physical component reported. \\
\hline Scarmeas et al. (2009) & $\begin{array}{l}\text { Physical activity: Time spent on a series of vigorous, moderate and light activities summed into an overall physical activity } \\
\text { score, which was then categorized into tertiles: no physical activity (used as reference), some physical activity and much } \\
\text { physical activity. }\end{array}$ \\
\hline Kivipelto et al. (2008) ${ }^{a}$ & Physical inactivity: Not participating in leisure time physical activity less than two times a week. \\
\hline Rovio et al. (2005) & Physical inactivity: Participating in leisure-time physical activity less than twice a week. \\
\hline Rovio et al. $(2007)^{a}$ & $\begin{array}{l}\text { Physical inactivity: Answering "no" to how many minutes do you walk, bicycle or have some other physical activity when } \\
\text { you are going to and from work? }\end{array}$ \\
\hline Anttila et al. (2002) & $\begin{array}{l}\text { Physical inactivity: Not holding one of the following 'sedentary' occupations: office work, intellectual work and service } \\
\text { branch work. }\end{array}$ \\
\hline Laurin et al. (2001) & $\begin{array}{l}\text { Physical activity: Engaging in exercise three or more times per week in an intensity greater than or equal to walking. } \\
\text { Physical inactivity: All other combinations of frequency and intensity. }\end{array}$ \\
\hline Yoshitake et al. (1995) & Physical inactivity: No daily exercise during the leisure period or moderate to severe physical activity at work. \\
\hline
\end{tabular}

${ }^{a}$ Studies not included in the reference list of the paper, but found by manual searching using author, year and dementia as a search term.

remove any mentions of sedentary behaviour and replace them with 'physical inactivity'. This would result in the conclusion that 'physical inactivity' is significantly associated with an increased risk of dementia, while the association of 'sedentary behaviour' with dementia still warrants further investigation. To address this gap, future studies should use the definition of sedentary behaviour supported by SBRN and use devices, e.g., activPAL inclinometer (http://www.palt.com) in conjunction with a subjective questionnaire to capture the context and specific types of sedentary behaviours. This will allow us to quantify the risk of sedentary behaviour on development of dementia in meta-analysis and also identify any differences in sedentary behaviour in people with and without dementia. Given the often-long time frame to develop dementia, interventions should consider using proxy measures such as cognitive function to assess their impact on health outcomes.

\section{Conflict of interest}

The authors declare no competing interests.

\section{Publisher's note}

Springer Nature remains neutral with regard to jurisdictional claims in published maps and institutional affiliations.

Received: 1 October 2020 Revised: 19 February 2021 Accepted: 3 March 2021

Published online: 30 March 2021

\footnotetext{
References

1. Yan, S. et al. Association between sedentary behavior and the risk of dementia: a systematic review and meta-analysis. Transl. Psychiatry 10, 112 (2020).
} 
2. Tremblay et al. Sedentary Behavior Research Network (SBRN) - Terminology Consensus Project process and outcome. Int. J. Behav. Nutr. Phys. Act. 14, 75 (2017).

3. WHO guidelines on physical activity and sedentary behaviour. https://www who.int/publications/i/item/9789240015128. Accessed 26 Mar 2021.

4. Lavie, C. J., Ozemek, C., Carbone, S., Katzmarzyk, P. T. \& Blair, S. N. Sedentary behavior, exercise, and cardiovascular health. Circ. Res. 124, 799-815 (2019).

5. Owen, N., Healy, G. N., Matthews, C. E. \& Dunstan, D. W. Too much sitting: the population health science of sedentary behavior. Exerc. Sport Sci. Rev. 38, 105-113 (2010)

6. Katzmarzyk, P. T. et al. Sedentary behavior and health: update from the 2018 Physical Activity Guidelines Advisory Committee. Med. Sci. Sports Exerc. 51, 1227-1241 (2019).
7. Lynch, B. M., Matthews, C. E. \& Wijndaele, K., Sedentary Behaviour Council of the International Society for Physical Activity and Health. New MeSH for sedentary behavior. J. Phys. Act. Health 16, 305 (2019).

8. Copeland et al. Sedentary time in older adults: a critical review of measurement, associations with health, and interventions. Br. J. Sports Med. 51, 1539 (2017).

9. Falck, R. S., Davis, J. C. \& Liu-Ambrose, T. What is the association between sedentary behaviour and cognitive function? A systematic review. Br. J. Sports Med. 51, 800-811 (2017).

10. Loprinzi, P. The effects of sedentary behavior on memory and markers of memory function: a systematic review. Phys. Sports Med. 47, 387-394 (2019).

11. Olanrewaju, O., Stockwell, S., Stubbs, B. \& Smith, L. Sedentary behaviours, cognitive function, and possible mechanisms in older adults: a systematic review. Aging Clin. Exp. Res. 32, 969-984 (2020). 